\title{
Teaching Quality Assessment System Based on Support Vector Machine Technology
}

\author{
https://doi.org/10.3991/ijet.v11i11.6251 \\ Wen-xue Huang, Xin Gao, Ning Wang, Yan-chao Yang, Ying Yan \\ North China University of Science and Technology
}

\begin{abstract}
A scientific, objective and accurate assessment of teaching quality is helpful in finding the relevant problems. So a highly accurate, quick and easy-to-implement teaching quality assessment system is necessary to build. The index value of the pulsed GTAW pool dynamic process by support vector machine inference and support vector machine neural networks is implemented in the teaching quality evaluation system. The teaching quality assessment system was tested on 30 teachers in a college. The results show that the assessment system is increasingly evidence-based. And the system can improve teaching quality and teaching management implementation.
\end{abstract}

Index Terms - teaching quality, evaluation system, support vector machine technology

\section{INTRODUCTION}

The reformation of education assessment undergoing revealed a new vision of assessment for teaching and learning, in which the Developmental Assessment Theory for Teaching and Learning (DATL) was applied and Information and Communication Technology was necessarily utilized. In this dissertation, the author paid serious attention on how to develop ICT platform to support the developmental assessment for teaching and learning[1-2]. As for this purpose, the author also focused on how to carry out developmental assessment, what' s support needed in carrying out developmental assessment. With the ICT platform dedicated for developmental assessment, the author also pointed out what' $s$ the tentative practice of developmental assessment said in test-bed schools.

With the development of internet and multimedia technology, web-based teaching is turning into an important teaching method day by day. As a key part of web-based teaching system, a good and effective teaching evaluating system is capable of testing teaching results, diagnosing teaching problems and formulating effective interactive state and feedback between teachers and students[3]. However, China' s web-based teaching evaluating systems currently are not perfect and many of them only offer none directional evaluating functions, for example the evaluation on the studying results of students. Moreover, a majority of studying evaluating systems adopts terminal assessment or the testing way through collecting students' behavior information based on their electronic records. Either those evaluating systems did not attach importance to the characteristics of web-based teaching or did not utilize the advantages of computer platform. What' s more, most of the teaching platforms currently did not offer a way for students to assess the teaching resources including teacher、 teaching contents、studying resource and studying supporting system evaluation. So, the educational managers have no way to find out the advantages and disadvantages of the teaching platform, which makes the enhancement of the teaching quality impossible.

With the deepening of China' $\mathrm{s}$ educational reform, teaching quality assessment which seen as an important link of operational mechanism of future education is being more and more valued by educators, develop and enhance teaching quality assessment has become an important and urgent problem. Establishing a scientific, objective, fair and practical teaching quality assessment system and method is an important content for colleges and universities9 teaching quality monitoring. Good works of the teaching quality assessment not only enhance teachers' teaching sense of responsibility, stimulate teachers9 enthusiasm and creativity, but also promote educational reform, guide teaching management, and provide the necessary basic data for the appointment and assessment of the colleges and universities5 jobs. However, because of heavy workload, complicated statistics and other reasons, colleges and universities often make the teaching quality assessment a mere formality, simple or one-sided. As the issue that difficult to implement educational assessment has been harassment for higher education development, the difficult to develop teaching quality assessment become an obstacle to improve teaching quality, it is necessary to seek a new scientific assessment method.

Multivariable linear regression and partial least-squares regression are limited to use in the above field because of their lack of ability to approach non-linear relationship. As a new technology, Artificial Neural Network has opened up a new avenue for pattern recognition, non-linear classification and artificial intelligence with its features of nonlinear mapping, learning classification and real-time optimization, which has been applied in the field of education assessment. However, Artificial Neural Network also has such disadvantages as massive amount of computation, easy to fall into local extreme points, weak extrapolation and lack ability of generalization [4].

Support Vector Machine is a new machine learning method proposed by Literature [5] based on statistical learning theory. The topology of Support Vector Machine is determined by the support vectors, compared with Artificial Neural Network which topology depends on the experience, it overcomes the limitation of high dimension, local extreme points and small samples effectively, and has the advantage of both Artificial Neural Network and gray model [6]. Through the learning of teaching quality assessment samples, the relationship between teaching quality and its influencing factors can been defined, and thus an objective and accurate assessment of a specific teaching quality approached. 
A scientific, objective and accurate assessment of teaching quality is helpful for detecting the relevant problems. It is also an important precondition for introducing improved measures and implementing teaching management. Traditional assessment methods of teaching quality have such disadvantages as being subjective, complex computation and weak effectiveness. Based on the analysis of various kinds of related methods, this paper puts forward a model for teaching quality assessment based on support vector machine. Theoretical and experimental results show that the model is effective in assessment, and compared with other methods, it is highly accurate, quick and easy to implement. The model is suitable for making teaching quality assessment in higher education.

\section{OVERVIEW}

The evaluation of teaching quality is a multi-level and multi-indicators problem. The evaluation system of teaching quality consists of seven first-class indicators, namely the quality of teachers $\theta_{1}$, teaching attitude $\theta_{2}$, teaching content $\theta_{3}$, teaching method $\theta_{4}$, teaching design $\theta_{5}$, teaching ability $\theta_{6}$ and teaching effectiveness $\theta_{7}$. Each indicator in the evaluation system is of different importance and includes a number of factors (or second-class indicators). The meaning expressed by the factors may have similar content, in other words, there is a certain relationship among the factors. So they are not individually independent [7-10].

1. The quality of teachers: standard of Putonghua, demeanor, instrument.

2. Teaching attitude: times of late, leave early and absences, status of counseling and marking students' duties, emphasis of students' feedback, attitude of give lessons.

3. Teaching content: scientifically, advancement and practicability of knowledge, training of learning abilities, penetration of emotional attitude and values.

4. Teaching methods: whether teaching objectives are clear or not, design of teaching strategies, design of teaching process.

5. Teaching methods: the choice and applications of multimedia teaching, guiding of learning methods, inspiring of teaching methods, and pertinence of organizing class.

6. Teaching ability: narrate the concept accurately, analyze the problem thoroughly, design blackboard writing reasonably, and operate experiment accurately.

7. Teaching effectiveness: status of students* feedback, status of student' testing, whether teaching goal is achieved or not.

\section{METHOD AND ALGORITHM}

In this section, we will discuss how to conduct the teaching quality evaluation system based on support vector machine technology. The main ideas of this paper are to implement the index value of the pulsed GTAW pool dynamic process by support vector machine inference and support vector machine neural networks. Structure of the proposed support vector machine is illustrated in Fig. 1.

As in illustrated in Fig.1, the support vector machine contains four layers: 1) Input nodes, 2) Membership function nodes, 3) Rules nodes and 4) Output nodes. Support vector machine is designed based on a series of IF-THEN

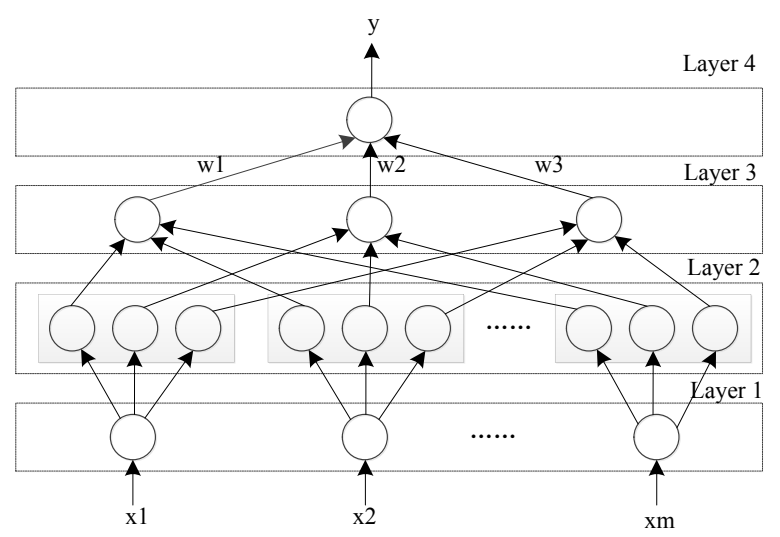

Figure 1. Structure of the proposed support vector machine

rules, which is used to describe the input/output mapping relationship:

$$
\begin{array}{r}
\text { Rule }(k) \text { :if } x_{1} \text { is } A_{1 k} \text { and } \\
x_{2} \text { is } A_{2 k} \mathrm{~L} \text { and } x_{n} \text { is } A_{n k} \\
\text { then } y=w_{k}, k \in\{1,2, \mathrm{~L}, N\}
\end{array}
$$

where $x_{i}$ and $y$ refer to the input variable and output variable, and the symbol $A_{i k}$ refers to the precondition part. Furthermore, $w_{k}$ means the output action strength that is corresponding to Rule $(k)$. Moreover, $N$ refers to the number fuzzy rules, and $n$ denotes the number of input variables. Based on the above definitions, the details of the support vector machine model are explained as follows [11].

\section{(1) Input nodes layer}

In this layer, each node is regarded as an input variable, and the node only can transmit input values to its neighbor and upper layer.

$$
v_{i}^{1}=x_{i}, i \in\{1,2, \mathrm{~L}, n\}
$$

\section{(2)Membership function nodes layer}

In the second layer, nodes should be divided to $\mathrm{N}$ clusters, and then each cluster should follow a fuzzy rule. In particular, each node of this layer is able to calculate the value of membership function. Assume that an external input is represented as $v_{i}^{1}$, and then the Gaussian membership function is described as followed.

$$
\begin{aligned}
& v_{i j}^{2}=\exp \left(-\frac{\left(v_{i}^{1}-m_{i j}\right)^{2}}{\delta_{i j}^{2}}\right), \\
& i \in\{1,2, \mathrm{~L}, n\}, j \in\{1,2, \mathrm{~L}, N\}
\end{aligned}
$$

where $m_{i j}$ and $\delta_{i j}$ refer to the centroid and the width of the Gaussian membership function respectively.

\section{(3)Rules nodes layer}

Different from the above layers, in this layer, the number of nodes means the number of fuzzy rules, and node in this layer is used to calculate the rule activation strength. Output values of this layer are listed in the following equation.

$$
v_{i j}^{3}=\prod_{i} v_{i j}^{2}, j \in\{1,2, \mathrm{~L}, N\}
$$

\section{(4)Output nodes layer}

Based on the above four layers, output values of this layer are computed as follows. 


$$
\begin{array}{r}
v^{4}=\frac{\sum_{j} v_{j}^{3} \cdot w_{j}}{\sum_{j} v_{j}^{3}} \\
v_{i}^{n+1}=w v_{i}^{n}+c_{1 i} \times r_{1} \times\left(P_{i}^{n}-X_{i}^{n}\right)+c_{2 i} \times r_{2}\left(G^{n}-X_{i}^{n}\right) \\
X_{i}^{n+1}=X_{i}^{n}+v_{i}^{n}
\end{array}
$$

so as to minimize the mean square error indicators:

$$
E(X)=\frac{1}{2 N} \sum_{p=1}^{n} \sum_{k=0}^{c}\left(Y_{k, p}(X)-t_{k, p}\right)
$$

Under the linear relationship, basic equation is shown in equation (2):

$$
\partial_{j}\left(e_{i j k l} \partial_{k} u_{l}-\eta_{k i j} \partial_{k} \varphi\right)=0(10)
$$

The linear differential equation can be expressed into the following simplified forms:

$$
\begin{aligned}
& L(\nabla, \omega) f(x, \omega)=0, \\
& \quad L(\nabla, \omega)=T(\nabla)+\omega^{2} \rho \mathrm{J}
\end{aligned}
$$

\section{EXPERIMENT RESULT}

Evaluation system is reasonable or not, the key lies $\mathrm{m}$ whether all factors can been considered comprehensively or not because this is the basis to guide students to score first-class indicators. Considering there is a certain degree of subjective arbitrariness in the scoring of each person, take average score of several students, thus this evaluation results are more authoritative and reliable. Based on the above evaluation system, the evaluation form is designed, and the teaching quality of 30 teachers in a college is investigated. The teaching quality assessment score by experts and students are listed in Table 1 . Take 1 to 25 samples as the learning samples, 26 to 30 samples as the test samples in the experiment.

Assessment Model. SVM model is divided into learning model and predictive model, the steps of modeling and prediction as follows:
Pretreat all samples and value them between 0 and 1; Establish SVM learning model by the use of learning samples and obtain Lagrange parameters difference value; Establish predictive model according to at-a] and b. Input predictive sample index and get the value of teaching quality evaluation. Hardware environment is Pentium (R) 4, 2.8 GHz CPU, 1.00 G RAM; Software environment is Matlab7. Value Penalty factor C 1000, s 0.004 , RBF parameter 820 .

In order to contrast prediction effectiveness, Multivariable linear regression model, partial least-squares regression model and Artificial Neural Network model are constructed. Take the relative error, mean square error MSE relative to predictive samples and time-consuming of CPU as comparative index.

(1) The experimental results. In order to contrast fitting results of the four models directly, fitting effect diagrams of learning samples and predictive samples are obtained, shown in Figure 2.

(2) Analysis of experimental results. The final predicted outcomes of the four models are shown in Table 2 (P-value represents predictive value, A-value represents actual value, and T-CPU represents time-consuming of CPU). As can be seen from Table 2, the predictive error of Multivariable linear regression is relatively big and its average relative error is more than $5.5 \%$. But the model is relatively simple, time-consuming of CPU is less. The prediction error of partial least-squares regression is biggest and its average relative error is more than $6 \%$. Because of the need for iterative solution algorithm, timeconsuming of $\mathrm{CPU}$ of partial least-squares regression is more.

There is one node in input layer which refers to teachers' number and sample selection table is shown as table 3 .

\begin{tabular}{|c|c|c|c|c|c|c|c|c|}
\hline \multirow{2}{*}{ Samples } & \multicolumn{7}{|c|}{ Indicator } & \multirow{2}{*}{$\mathbf{y}$} \\
\hline & $\theta 1$ & $\theta 2$ & $\theta 3$ & $\theta 4$ & $\theta 5$ & $\theta 6$ & $\theta 7$ & \\
\hline 1 & 6.607 & 3.368 & 4.746 & 4.756 & 2.232 & 4.318 & 6.161 & 7.219 \\
\hline 2 & 7.221 & 5.419 & 6.472 & 3.218 & 4.377 & 6.154 & 7.746 & 7.257 \\
\hline 3 & 8.375 & 6.601 & 7.801 & 5.329 & 5.301 & 5.981 & 6.077 & 8.605 \\
\hline 4 & 8.299 & 8.338 & 6.721 & 8.196 & 6.504 & 7.808 & 6.479 & 7.862 \\
\hline 5 & 8.306 & 8.159 & 5.441 & 9.350 & 7.703 & 9.357 & 8.152 & 7.529 \\
\hline
\end{tabular}
Network is completed after 7638 training and the training performance is shown as figure 2 . The trained neural network is used to predict latter three years' data and the

\begin{tabular}{|c|c|c|c|c|c|c|c|c|}
\hline \multirow{2}{*}{ Samples } & \multicolumn{2}{|c|}{ MLR model } & \multicolumn{2}{|c|}{ PLSR model } & \multicolumn{2}{|c|}{ ANN model } & \multicolumn{2}{|c|}{ SWM model } \\
\hline & P-value & A-value & P-value & A-value & P-value & A-value & P-value & A-value \\
\hline 26 & 7.393 & 7.72 & 7.540 & 9.87 & 6.837 & 0.38 & 6.679 & 1.37 \\
\hline 27 & 6.883 & 5.01 & 6.964 & 3.89 & 6.815 & 5.95 & 7.044 & 2.78 \\
\hline 28 & 6.955 & 6.82 & 7.314 & 2.01 & 7.148 & 4.24 & 7.356 & 1.45 \\
\hline 29 & 8.988 & 4.44 & 8.759 & 6.88 & 9.868 & 4.91 & 9.075 & 3.52 \\
\hline 30 & 7.886 & 5.52 & 7.813 & 6.39 & 7.906 & 5.28 & 8.388 & 0.48 \\
\hline MSE & \multicolumn{2}{|c|}{0.172} & \multicolumn{2}{|c|}{0.195} & \multicolumn{2}{|c|}{0.139} & \multicolumn{2}{|c|}{0.006} \\
\hline T-CPU & \multicolumn{2}{|c|}{0.016} & \multicolumn{2}{|c|}{0.859} & \multicolumn{2}{|c|}{2.891} & \multicolumn{2}{|c|}{0.234} \\
\hline
\end{tabular}
results are shown as table 4 and figure 3 .

TABLE I.

EVALUATION INDICATOR AND EVALUATION RESULTS

TABLE II.

PREDiCTED RESUlts OF THE Four MODELS 
PAPER

TeAching QuAlity Assessment System BASED on Support Vector Machine TeChnOlogy
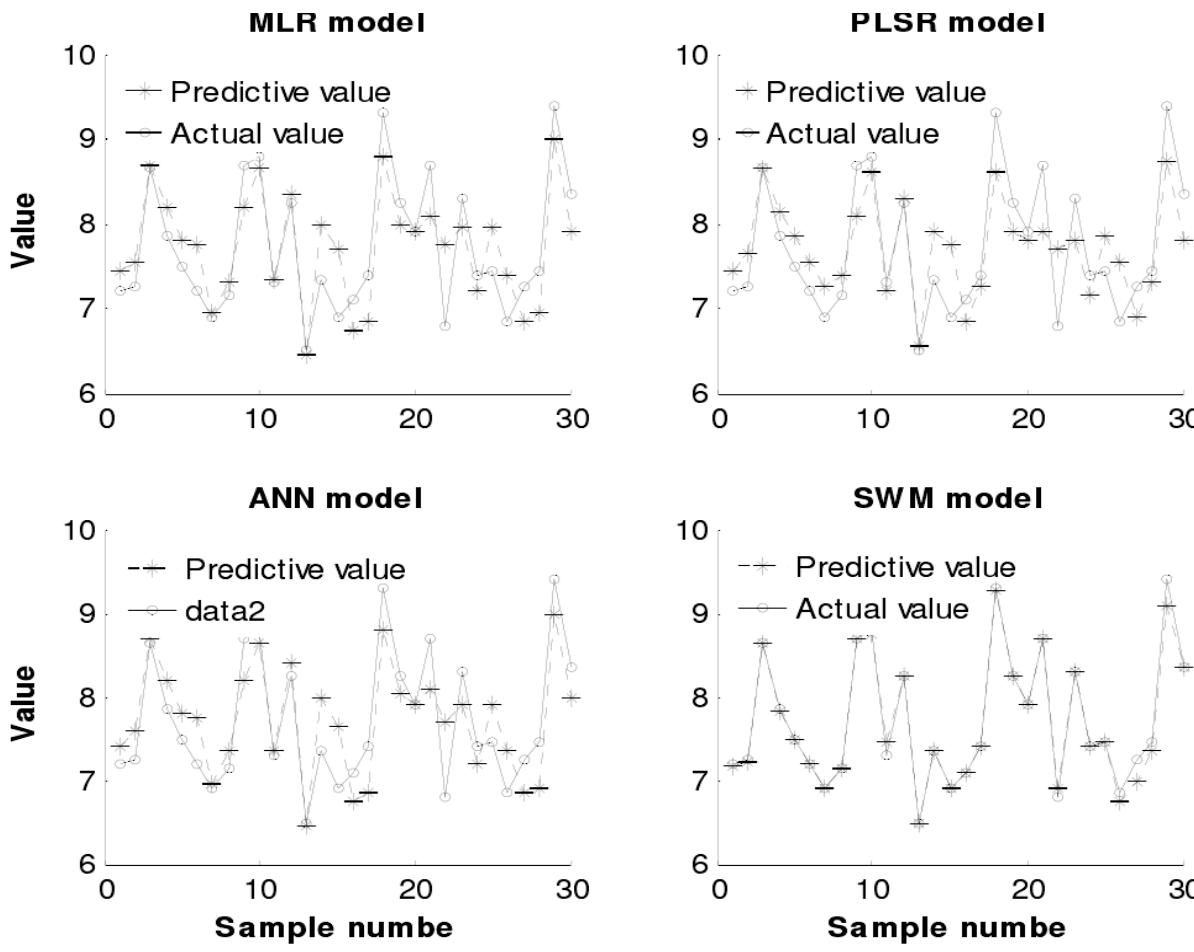

SWM model

10

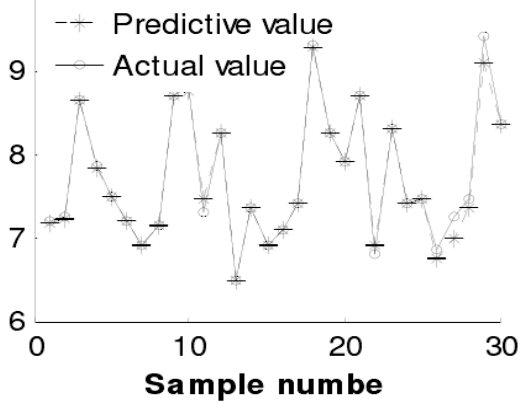

Figure 2. Fitting effect of the four models

TABLE III. SAMPLE SELECTION TABLE

\begin{tabular}{|c|c|c|c|c|c|c|c|c|}
\hline & NO. & Year & V Value & Serving person & Per capita disposable & Income & G-Markov prediction results & Teaching person \\
\hline \multirow{10}{*}{ Training sample } & 1 & 2000 & 118.56 & 168.81 & 4283.1 & 1375.7 & 6.29 & 6.23 \\
\hline & 2 & 2001 & 127.84 & 179.23 & 4838.9 & 1638.4 & 6.12 & 6.41 \\
\hline & 3 & 2002 & 169.82 & 184.35 & 5160.3 & 2112.0 & 6.54 & 6.45 \\
\hline & 4 & 2003 & 176.53 & 188.61 & 5425.2 & 2391.3 & 6.79 & 6.96 \\
\hline & 5 & 2004 & 180.92 & 192.05 & 5854.0 & 2831.9 & 7.05 & 7.20 \\
\hline & 6 & 2005 & 187.05 & 198.23 & 6280.1 & 3175.4 & 7.28 & 7.46 \\
\hline & 7 & 2006 & 334.53 & 201.60 & 6859.6 & 3522.5 & 8.01 & 7.85 \\
\hline & 8 & 2007 & 345.71 & 209.75 & 7702.1 & 3878.6 & 8.72 & 8.79 \\
\hline & 9 & 2008 & 358.36 & 216.04 & 8472.2 & 3442.3 & 9.66 & 9.93 \\
\hline & 10 & 2009 & 360.02 & 227.28 & 9421.6 & 4710.7 & 11.27 & 11.03 \\
\hline \multirow{3}{*}{ Test sample } & 1 & 2010 & 373.45 & 216.39 & 10493.5 & 5285.8 & 12.41 & 12.13 \\
\hline & 2 & 2011 & 386.08 & 241.43 & 11759.2 & 6229.8 & 13.63 & 13.65 \\
\hline & 3 & 2012 & 400.81 & 250.87 & 13785.8 & 7770.6 & 15.89 & 16.17 \\
\hline
\end{tabular}

TABle IV. Prediction Value and Relative ERror Table of Single Model from 2000 to 2012

\begin{tabular}{|c|c|c|c|c|c|}
\hline Year & Actual Value & G-Markov model & Single-variable prediction value & Multiple-variable prediction value & Improved model \\
\hline 2000 & 6.94 & 6.78 & 7.21 & 6.79 & 6.75 \\
\hline 2001 & 7.21 & 7.03 & 7.16 & 7.28 & 6.96 \\
\hline 2002 & 7.44 & 7.25 & 7.22 & 7.44 & 7.51 \\
\hline 2003 & 7.84 & 8.01 & 7.92 & 8.05 & 7.92 \\
\hline 2004 & 8.76 & 8.78 & 8.23 & 8.69 & 9.04 \\
\hline 2005 & 9.83 & 9.65 & 9.69 & 9.19 & 9.69 \\
\hline 2006 & 11.01 & 11.28 & 11.01 & 11.25 & 10.99 \\
\hline 2007 & 12.11 & 12.41 & 12.00 & 12.01 & 11.92 \\
\hline 2008 & 13.94 & 13.62 & 13.97 & 14.08 & 14.18 \\
\hline 2009 & 16.10 & 15.87 & 16.10 & 16.04 & 16.01 \\
\hline 2010 & 17.12 & 17.44 & 16.21 & 17.13 & 17.22 \\
\hline MAD & & 0.3632 & 0.1801 & 0.2425 & 0.1834 \\
\hline MSE & & 0.5098 & 0.0956 & 0.1311 & 0.4992 \\
\hline MAPE & & 2.5235 & 1.5225 & 0.1950 & 1.5969 \\
\hline
\end{tabular}




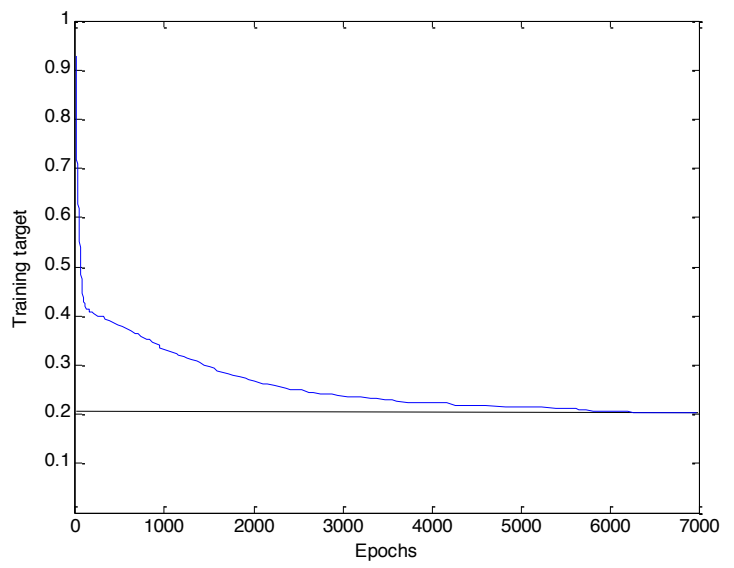

Figure 3. Performance training curve

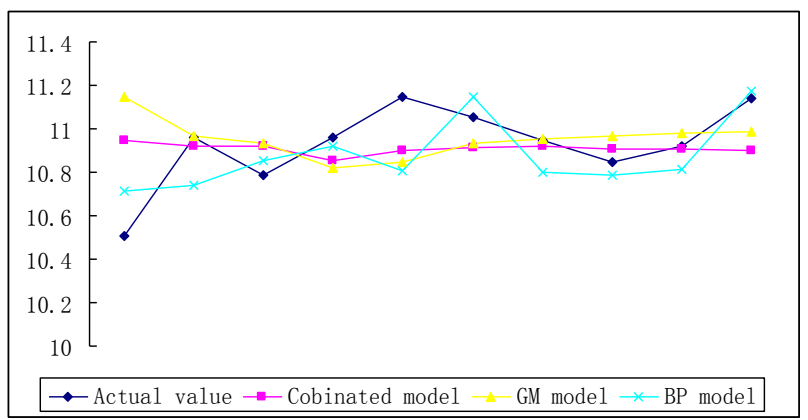

Figure 4. Comparison of prediction value of three models to actual test value

\section{DISCUSSION}

Artificial Neural Network has the ability to approximate non-linear functions and improve the solution accuracy of the non-linear function, but its shortcomings are obvious: first, complex solution and more time-consuming. It can be seen from Table 2 that Artificial Neural Network takes 2.9 seconds, which more 100 times than the multiple linear model, more 3 times than the partial least-squares regression model and 12 times than the SVM model respectively. Second, the model is unstable. When the Artificial Neural Network model search for local optimal solution excellent, the results are not the only, and table 2 is a particular prediction.

It can been see from Table 2 that SVM model has higher prediction accuracy with the average relative error less than $2 \%$, and the time-consuming of CPU is little, only $0.234 \mathrm{~s}$. This is because the SVM model is based on structural risk minimization which is a compromise taking into account learning complexity and model accuracy, makes the model have better generalization ability to improve the prediction accuracy for unknown samples; SVM topology is determined by a small number of support vector makes the model simple, faster solution speed. Because SWN is for the global optimization, the obtained solution is the only optimal one.

\section{CONCLUSION}

The establishment of teaching quality assessment system makes evaluation of teachers increasingly evidencebased. However, the quantitative non-linear function relationship between teaching quality and its influencing fac- tors is difficult to express, so there is a big one-sidedness and subjectivity in the final given evaluation scores, and objectivity and fair of evaluation results is affected. SVM learning model has the ability of approaching non-linear relationship, and can find dependency of teaching quality on evaluation index through the learning of some given samples. Experimental results show that the model is effective in assessment, highly accurate, quick and easy to implement. It is of significant theoretical and application value for the model to find class teaching problems, improve teaching quality and implement teaching management.

\section{REFERENCES}

[1] J. Yang, S. He, Y. Lin, et al., "Multimedia cloud transmission and storage system based on internet of things," Multimedia Tools and Applications, pp. 1-16, October 2015. https://doi.org/10.1007/ s11042-015-2967-9

[2] H. Jing, "The Study on the Impact of Data Storage from Accounting Information Processing Procedure," International Journal of Database Theory and Application, vol. 8, no.3, pp. 323-332, June 2015. https://doi.org/10.14257/ijdta.2015.8.3.28

[3] A. Drigas, and M. Karyotaki, "Online and other ICT-based Training Tools for Problem-solving Skills," International Journal of Emerging Technologies in Learning, vol. 11, no.6, pp. 39-44, June 2016. https://doi.org/10.3991/ijet.v11i06.5340

[4] H. Loussifi, K. Nouri, and N.B. Braiek, "A New Efficient Hybrid Intelligent Method for Nonlinear Dynamical Systems Identification: The Wavelet Kernel Fuzzy Neural Network," Communications in Nonlinear Science and Numerical Simulation, no. 32, pp.10-30, 2016. $\quad$ https://doi.org/10.1016/j.cnsns.2015. 08.010

[5] A. Anastassiou George, and F.A. Iatan Iuliana, "Recurrent Neural Fuzzy Network," Journal of Computational Analysis and Applications, vol.20, no.2, pp.213-225, 2016.

[6] C. Xu, Q. Zhang, and Y. Wu, "Existence and Exponential Stability of Periodic Solution to Fuzzy Cellular Neural Networks with Distributed Delays," International Journal of Fuzzy Systems, vol. 18, no.1, pp. 41-51, 2016. https://doi.org/10.1007/s40815-015-0103-7

[7] C. Zheng, X.Zhang, and Z. Wang, "Mode and Delay-dependent Stochastic Stability Conditions of Fuzzy Neural Networks with Markovian Jump Parameters," Neural Processing Letters, vol. 43, no.1, pp. 195-217, 2016. https://doi.org/10.1007/s11063-0159413-X

[8] H.C. Nejad, M. Farshad, O. Khayat, et al., "Performance Verification of a Fuzzy Wavelet Neural Network in the First Order Partial Derivative Approximation of Nonlinear Functions," Neural Processing Letters, vol. 43, no.1, pp. 219-230, 2016. https://doi.org/10.1007/s11063-015-9414-9

[9] A.Wu, and Z. Zeng, "Boundedness, Mittag-Leffler Stability and Asymptotical Omega-Periodicity of Fractional-Order Fuzzy Neural Networks," Neural Networks, no. 74, pp. 73-84, 2016. https://doi.org/10.1016/j.neunet.2015.11.003

[10] R. Wai, M. Chen, and J. Yao, "Observer-Based Adaptive FuzzyNeural-Network Control for Hybrid Maglev Transportation System," Neurocomputing, no. 175, pp.10-24, 2016. https://doi.org/10.1016/j.neucom.2015.10.006

[11] Z. Ma, G. Sun, D. Liu, et al., "Dissipativity Analysis for DiscreteTime Fuzzy Neural Networks with Leakage and Time-Varying Delays," Neurocomputing, no.175, pp. 579-584. 2016. https://doi.org/10.1016/j.neucom.2015.10.098

\section{AUTHORS}

Wen-xue Huang, Xin Gao, Ning Wang, Yan-chao Yang, and Ying Yan are with North China University of Science and Technology (huangwenxue03@163.com).

Submitted 09 September 2016. Published as resubmitted by the authors 23 October 2016. 